\title{
Lidil
}

Revue de linguistique et de didactique des langues

$63 \mid 2021$

Littératie numérique et didactique des langues et des cultures

\section{La macrostructure comme critère d'évaluation de la qualité de notes prises par des étudiants universitaires dans les modes manuscrit et numérique}

Macrostructure as a Criterion to Evaluate the Quality of Notes Taken by

University Students in the Manuscript and Digital Modes

Audrey Pépin et Odette Gagnon

\section{OpenEdition}

Édition électronique

URL : http://journals.openedition.org/lidil/8683

DOI : $10.4000 /$ lidil.8683

ISSN : 1960-6052

Éditeur

UGA Éditions/Université Grenoble Alpes

Édition imprimée

ISBN : 978-2-37747-283-3

ISSN : 1146-6480

Référence électronique

Audrey Pépin et Odette Gagnon, « La macrostructure comme critère d'évaluation de la qualité de notes prises par des étudiants universitaires dans les modes manuscrit et numérique », Lidil [En ligne], 63 | 2021, mis en ligne le 30 avril 2021, consulté le 30 avril 2021. URL : http://journals.openedition.org/ lidil/8683; DOI : https://doi.org/10.4000/lidil.8683

Ce document a été généré automatiquement le 30 avril 2021.

(C) Lidil 


\title{
La macrostructure comme critère d'évaluation de la qualité de notes prises par des étudiants universitaires dans les modes manuscrit et numérique
}

\author{
Macrostructure as a Criterion to Evaluate the Quality of Notes Taken by \\ University Students in the Manuscript and Digital Modes
}

Audrey Pépin et Odette Gagnon

\section{Introduction}

1 L'intérêt pour un usage pédagogique de la technologie ne cesse de grandir depuis l'adoption du «Plan d'action numérique en éducation et en enseignement » en 2018 (Gouvernement du Québec, 2018). Ce plan mise sur l'intégration efficace du numérique en classe de sorte que les différents acteurs du milieu scolaire soient amenés à développer leurs compétences numériques. Il y a donc fort à parier que le numérique est et sera de plus en plus utilisé en classe pour la réalisation de diverses activités, dont certainement la prise de notes (PDN).

2 Le point de départ de la réflexion ayant mené à la recherche ${ }^{1}$ (Pépin, 2020) à laquelle nous nous référons dans cet article trouve son origine dans les nombreuses questions ayant émergé d'une recherche exploratoire menée à la suite de l'intégration de la tablette numérique à l'ensemble du Séminaire de Chicoutimi (école secondaire) en 2014 pour la réalisation des diverses activités de la classe, dont la PDN : Comment organiser la PDN avec une tablette? Quels sont les effets de la tablette sur les habitudes de PDN? (Pépin et coll., 2017). Cette recherche exploratoire avait permis de faire ressortir les similitudes et les différences entre les deux modes de PDN (numérique et manuscrit) 
quant à l'organisation du contenu des notes, à la qualité de la langue et à l'utilisation de procédés linguistiques de réduction (ibid.), mais elle n'avait pas permis d'identifier clairement les effets que la tablette (ou le mode numérique) pouvait avoir sur la PDN ou, plus précisément, sur les habitudes de PDN.

La présente recherche visait donc à combler cette lacune et à observer les habitudes de PDN des apprenants lors de leurs PDN manuscrites ou numériques (à l'aide d'une tablette ou d'un ordinateur). Cependant, elle a été menée auprès d'apprenants de niveau universitaire, notamment parce qu'ils sont susceptibles de prendre une plus grande quantité de notes étant donné l'enseignement magistral qui prévaut dans les pratiques pédagogiques universitaires (Gohier et coll., 2005; Prégent et coll., 2009). L'enseignement magistral exige qu'ils prennent des notes pour maintenir leur attention (Chamberland et coll., 2011) et pour traiter la grande quantité d'informations nouvelles auxquelles ils sont exposés (Pollet, 2001).

\section{Problématique}

En réalité, l'intégration du numérique vient transformer la relation bipartite entre l'enseignant et l'étudiant en relation tripartite dans laquelle l'élément qui s'ajoute est la technologie. Cette relation tripartite se subdivise en quatre types d'interaction: (1) enseignant-technologie, (2) enseignant-étudiant, (3) étudiant-technologie et (4) étudiant-étudiant (Li \& Pow, 2011, p. 320). Dans notre cas, c'est l'interaction entre l'étudiant et la technologie (ibid.) qui nous intéresse plus particulièrement, c'est-à-dire le fait que la technologie peut avoir une incidence sur les habitudes de PDN d'un noteur. En fait, c'est plutôt l'affordance du mode numérique ou, en d'autres termes, les fonctionnalités spécifiques qu'offre l'outil numérique à son utilisateur, et non la technologie en elle-même, qui a une incidence sur les habitudes de PDN d'un noteur (ibid.). C'est donc les fonctionnalités offertes par les différents outils (technologie vs papier et crayon) qui amènent le noteur à réaliser ses notes différemment Ces deux modes comportent des avantages et des inconvénients liés à leur affordance, ce à quoi quelques études se sont intéressées.

5 Les études réalisées par Bui et coll. (2013), Fiorella et Mayer (2017), Luo et coll. (2018), Mueller et Oppenheimer (2014) révèlent que les notes prises en mode numérique seraient plus complètes que celles prises de façon manuscrite, notamment en raison de la vitesse supérieure de l'écriture (Aragón-Mendizábal et coll., 2016; Stacy \& Cain, 2015), qui permet de noter plus d'informations. La PDN numérique comporte par ailleurs d'autres avantages non négligeables, comme la meilleure lisibilité des notes et la possibilité de lancer une recherche dans les notes pour y retrouver rapidement une information précise (Stacy \& Cain, 2015, p. 5). La PDN numérique peut aussi permettre au noteur de partager ses notes ou de collaborer avec d'autres personnes pour les réaliser. En effet, avec la culture du Web 2.0, «Internet se caractérise par le “partage" de l'information et l'“ouverture" » (Rinck \& Mansour, 2013, p. 621).

6 Il est donc indéniable que la PDN numérique présente des avantages non négligeables. Toutefois, selon les études menées par Bui et coll. (2013), Fiorella et Mayer (2017), Luo et coll. (2018), Mueller et Oppenheimer (2014), les notes numériques comportent beaucoup plus de segments de "verbatim ", c'est-à-dire de segments d'informations transcrits comme entendus (à l'instar d'une dictée). Or, une telle façon de faire ne permettrait qu'un traitement superficiel des informations (mémoire à court terme), 
menant à une moindre compréhension et rétention de ces dernières. De plus, le fait de taper les notes plutôt que de les écrire à la main aurait un impact négatif sur l'encodage des informations. En effet, la neuroimagerie a révélé que taper chaque lettre fait appel à la même commande motrice générique, ce qui résulte en un moindre encodage des informations dans la mémoire (Longcamp et coll., 2008). Enfin, l'outil numérique utilisé pour la PDN pourrait être une source de distraction pour le noteur, ce qui nuirait à sa PDN (Stacy \& Cain, 2015, p. 3).

7 En ce qui concerne la PDN manuscrite, l'écriture manuscrite, puisqu'elle est plus lente que l'écriture numérique, permettrait aux noteurs de mieux comprendre les informations qu'ils notent. C'est pourquoi plusieurs étudiants préfèrent l'écriture manuscrite à l'écriture numérique (Peverly \& Sumowski, 2012, p. 115). De plus, les contraintes psychomotrices de l'écriture manuscrite ralentiraient la transcription des informations et obligeraient alors les noteurs à sélectionner encore mieux les informations pertinentes et à mieux les reformuler que lors de la PDN numérique, ce qui favoriserait un meilleur traitement de l'information (Manzi et coll., 2017) ou un encodage plus profond (mémoire à long terme) des informations (Aragón-Mendizábal et coll., 2016). La neuroimagerie a également révélé que le tracé de chaque lettre produit une signature neurale différente dans le cortex moteur, ce qui permet aussi un meilleur encodage des informations dans la mémoire (Longcamp et coll., 2008). Par contre, l'écriture manuscrite étant lente, elle ne permettrait pas au noteur de noter autant d'informations que s'il les tapait.

Bref, les fonctionnalités différentes de chacun des modes font en sorte que chacun a des avantages et des inconvénients. On peut donc présumer que les noteurs seront amenés à prendre leurs notes différemment selon le mode.

9 Par ailleurs, nous constatons que les études ayant comparé les deux modes de PDN se sont surtout attachées au processus de PDN, et non aux notes elles-mêmes. Pourtant, si l'on veut évaluer l'effet des PDN réalisées dans un mode ou l'autre sur les apprentissages qui en découlent, il faut être en mesure d'évaluer la qualité des notes. La question qui se pose alors est de savoir comment nous pouvons évaluer la qualité de notes.

Considérant que prendre des notes revient à opérer une réduction dans les informations "sources", cette réduction se manifestant au niveau conceptuel et au niveau formel (Bouchet et coll., 2014 ; Piolat, 2001 ; Roussey \& Piolat, 2003), il faut voir comment cette « réduction » doit se présenter dans les notes pour que celles-ci soient considérées comme étant de qualité et, ainsi, susceptibles d'avoir un effet positif sur les apprentissages. Selon Williams et Eggert (2002), « les notes efficaces sont caractérisées par une organisation claire [des informations] (c'est-à-dire que les relations entre les idées principales et secondaires sont clairement définies)» (p.177, notre traduction). Williams et Eggert (2002) ont aussi mentionné que les notes les plus efficaces sont celles dans lesquelles la structure générale de l'exposé ou du discours source (DS) est mise en évidence. Autrement dit, des notes de qualité, et par conséquent efficaces, sont des notes qui, par l'organisation claire de leur contenu et par la présence des informations essentielles du DS, reflètent la macrostructure (MS) de ce DS. Pour évaluer la qualité des notes, il faut donc être en mesure d'observer si les informations essentielles du DS sont présentes dans les notes et si leur organisation permet de bien mettre en évidence la MS du DS. Notre hypothèse serait que des notes de qualité permettraient aux 
apprenants de mieux réussir à un examen. Les objectifs de la recherche s'énoncent donc comme suit :

1. Concevoir une grille pour évaluer la qualité des notes ${ }^{2}$.

À partir de cette grille :

1.1. Vérifier s'il y a une différence quant à l'organisation et à la sélection du contenu des notes réalisées dans les deux modes.

1.1.1. Relever les aspects de l'organisation et de la sélection du contenu qui diffèrent entre les modes.

1.2. Mettre les indices quant à l'organisation du contenu et à la sélection de ce contenu en parallèle avec un résultat à un examen afin d'observer leur incidence sur la performance, et ce, dans les deux modes.

11 La prochaine section sera consacrée au premier objectif de recherche, soit à la présentation de la grille d'analyse qui a été créée à partir d'une recension d'écrits. La section 4 , quant à elle, sera consacrée à la présentation de la méthodologie de notre recherche. Les sections 5 et 6 seront consacrées à la présentation des résultats et à la discussion.

\section{Grille d'analyse de la qualité des notes}

Pour évaluer la qualité des notes, nous avons conçu une grille ${ }^{3}$ d'analyse à partir d'une recension des écrits. Rappelons que la qualité des notes s'actualise par la capacité du noteur à faire ressortir ou même à transposer la MS d'un DS dans ses notes (Williams \& Eggert, 2002). Notre grille (voir annexe I : grille d'analyse de la qualité des notes) prend donc en compte la MS du DS et la MS des notes, ce qui rend possible une mise en parallèle entre les deux.

De cette recension des écrits sont ressortis deux types de procédés permettant au noteur de transposer la MS d'un DS dans ses notes: les procédés de réduction et de hiérarchisation des informations. Avant de présenter ces procédés, il n'est pas inutile d'insister sur le fait que leur mise en œuvre est très intimement liée à la compréhension qu'a le noteur des informations du DS. En effet, le noteur ne sera en mesure de réduire et de hiérarchiser les informations du DS, et de les organiser sous la forme d'une "macrostructure [...] comport[ant] les informations les plus importantes du message » (Piolat, 2001, p. 23) que s'il les comprend (Cody et coll., 2016).

Puisque les procédés de réduction et de hiérarchisation permettent au noteur de transposer la MS d'un DS dans ses notes, ils sont évidemment pris en compte dans notre grille.

\subsection{Réduction des informations}

Étant donné que la cadence de la parole est supérieure à celle de l'écriture, la PDN lors de l'écoute, dans un cours universitaire, par exemple, se déroule dans l'urgence (Piolat, 
2010, p. 55). Pour être capable de transposer la MS du DS vers ses notes et ainsi de prendre des notes de qualité dans ce cadre temporel limité (Peverly \& Sumowski, 2012, p. 104), le noteur doit réduire les informations du DS: au niveau conceptuel, en sélectionnant les informations principales; au niveau formel, en laissant le moins de traces graphiques possible.

16 Au niveau conceptuel, le noteur doit sélectionner les informations principales et les raccourcir, en appliquant des macrorègles sémantiques de réduction afin d'obtenir des macropropositions qui représentent le sens global du discours (Van Dijk \& Kintsch,1983, p.189-191). Ces macrorègles qui permettent de dériver la MS d'un DS vers les notes sont la suppression, la généralisation et la construction ${ }^{4}$. Ces dernières sont prises en compte dans notre grille (colonne F). Par ailleurs, d'autres éléments pourraient avoir une incidence plus ou moins positive sur la sélection des informations opérée par le noteur et sont pris en compte (colonne A) : les marqueurs de scripturalité qui incitent à la PDN (ex.: l'enseignant signifie que ce qu'il dit est important ou l'enseignant inscrit une information au tableau) et les marqueurs d'oralité qui inhibent plutôt la PDN (ex.: un étudiant pose une question ou une discussion est amorcée entre les étudiants) (Boch, 2000, p. 140-144).

$\mathrm{Au}$ niveau formel, pour accélérer sa PDN, le noteur doit essayer de limiter ses traces graphiques en agissant sur le lexique et la syntaxe. Sur le plan lexical, il peut opter pour des procédés de resserrement (Piolat, 2010, p.52) nommés aussi outils de condensation par Branca-Rosoff (1998, p. 288) ou procédés de condensation par Faraco et coll. (2003, p. 99). Parmi ces procédés de resserrement ou de condensation, il y a les procédés abréviatifs qui consistent à raccourcir des mots par l'omission de lettres (Piolat, 2001, p. 62). Les abréviations employées peuvent être dites conventionnelles ou créées de toute pièce par la troncature de la terminaison ou par la conservation de l'amorce (par exemple Branca-Rosoff, 1998, p. 288-293 ; Piolat, 2001, p. 66-69). Il y a aussi les procédés substitutifs qui consistent à employer des symboles logiques $(+,=,-$, etc.), iconiques $(\downarrow, \uparrow, !$, etc.) et grécoalphabétiques $(\Psi, \pi$, etc.) au lieu de certains mots (Piolat, 2001, 2010). Ces symboles peuvent représenter une idée ou être utilisés de façon analogique. Sur le plan syntaxique, le noteur peut employer le style télégraphique, c'est-à-dire abandonner la syntaxe et la linéarité de l'écriture pour écrire des mots-clés ou des idées dans des listes à puces, par exemple (Piolat \& Boch, 2004). De plus, les procédés substitutifs peuvent être employés pour remplacer certains éléments de la syntaxe habituelle (Piolat \& Boch, 2004).

\subsection{Hiérarchisation des informations}

Pour prendre des notes de qualité, en plus de réduire les informations qu'il note dans le but de lutter contre l'urgence, le noteur doit aussi attribuer un niveau hiérarchique à chacune des informations qu'il sélectionne de façon à rendre apparente la structure hiérarchique du DS dans ses notes. Le modèle présenté par Faraco et coll. (2003) distingue quatre niveaux hiérarchiques et suppose que les informations de niveau différent ne devraient pas être présentées de la même façon dans les notes :

1.1. Unités majeures (grands thèmes) : séparation de ces unités par des connecteurs et spécification par des titres-résumés. 


\begin{tabular}{|l|l|l|l|}
\hline & 1.1.1. & \multicolumn{2}{l|}{$\begin{array}{l}\text { Unités conceptuelles : séparation de ces unités par des connecteurs et spécification } \\
\text { par des thèmes-titres (sorte de sous-titres). }\end{array}$} \\
\hline & & 1.1.1.1. & $\begin{array}{l}\text { Unités de sens de base (apport d'une seule information) : syntagme ou } \\
\text { groupe de phrases. }\end{array}$ \\
\hline & & 1.1.1.1.1. & $\begin{array}{l}\text { Sous-unités de base (information qui complète unité de } \\
\text { base) : syntagme ou phrase complexe. }\end{array}$ \\
\hline
\end{tabular}

19 Le noteur peut user d'autres stratégies que celle présentée dans Faraco et coll. (2003) pour marquer la hiérarchie entre les informations plus globales et ainsi faire ressortir la MS, par exemple des titres et des intertitres (Rebeyrolle, Jacques \& PéryWoodley, 2009).

D'autres procédés encore peuvent être utilisés, comme l'emploi du style spatiotélégraphique qui par son incidence sur «l'organisation syntaxico-sémantique » et sur « la mise en forme de l'espace graphique» (Piolat, 2001, p. 75) fait ressortir la hiérarchisation entre les informations (Branca-Rosoff, 1998). Enfin, le noteur peut aussi faire ressortir la hiérarchisation en utilisant des marques de métadiscours, c'est-à-dire en ajoutant des symboles ou des commentaires pour indiquer l'importance d'un passage, par exemple, et en mettant en exergue certaines informations (soulignement, encadrement, emploi de lettres majuscules, etc.).

21 En résumé, ces procédés de réduction et de hiérarchisation des informations, dont la mise en œuvre atteste de la compréhension du message, permettent au noteur de prendre des notes de qualité dans lesquelles la MS du DS est transposée. C'est pourquoi ils ont été retenus comme critères d'analyse de la qualité des notes dans notre grille.

\section{Méthodologie}

Notre recherche consiste à analyser la MS de notes prises dans les deux modes, manuscrit et numérique, à l'aide de notre grille.

Pour ce faire, nous avons constitué un corpus de 22 documents de notes, soit 12 manuscrits et 10 numériques. Ces documents ont été réalisés par des étudiants universitaires volontaires de première année inscrits au baccalauréat ${ }^{6}$ en éducation préscolaire et en enseignement primaire ou au baccalauréat en enseignement et en adaptation scolaire lors de deux séances du cours Apprentissage de la lecture et de l'écriture. Il est important de spécifier que ces étudiants ont pris leurs notes dans le mode dans lequel ils étaient à l'aise, notre but étant d'observer les habitudes de PDN en contexte authentique.

Nous avons assisté aux deux séances de cours ciblées afin d'identifier et de hiérarchiser les unités de sens du discours source en nous inspirant du modèle hiérarchique présenté à la section précédente. En d'autres termes, nous devions nous-mêmes extraire la MS du DS pour être en mesure d'identifier la MS dans les documents de notes et, par la suite, de mettre les deux en parallèle afin d'évaluer la qualité des notes.

Il s'est toutefois avéré que les étudiants n'ont pas pris beaucoup de notes durant les deux séances de cours ciblées. En effet, contrairement à ce que nous aurions souhaité, ces deux séances comportaient des ateliers pratiques au cours desquelles les étudiants 
n'avaient pas à prendre de notes. De plus, ils ont eu accès à des documents de référence comportant une bonne partie des notions à l'étude, et celles-ci y étaient présentées de façon organisée (MS) pour justement leur éviter de prendre trop de notes.

Comme les participants ont eu accès à une bonne partie de la MS par l'entremise des documents de référence, nous ne pouvions pas utiliser la grille telle que nous l'avions conçue initialement pour évaluer la capacité des noteurs à produire des notes de qualité par la compréhension et la transposition de la MS du DS dans les notes. Nous avons dû modifier notre grille de manière à tenir compte du fait que les cours s'appuyaient sur des documents de référence présentant une partie de la MS du DS 7 . Cette nouvelle grille permettait notamment d'identifier les documents de référence ayant permis d'obtenir, dans certains cas, le découpage hiérarchique du $\mathrm{DS}^{8}$. De cette façon, il était possible de noter la présence ou non dans les notes des informations du DS et ainsi de constater la sélection du contenu opérée par le noteur. Nous avons également conservé tous les procédés permettant aux noteurs d'organiser leurs notes. Nous avons toutefois décidé de ne pas conserver les colonnes à propos des abréviations et des icônes substitutives, car leur analyse aurait dépassé le cadre d'un mémoire de maitrise (voir annexe II : grille d'analyse de la qualité des notes modifiée).

La grille modifiée nous a permis, d'une part, de faire une analyse descriptive de l'organisation des notes. D'autre part, le découpage des informations du discours source et des notes en unités de sens nous a permis de procéder à des analyses quantitatives du contenu des notes, et ce, pour les notes réalisées dans les deux modes (objectifs 1.1 et 1.1.1). De plus, comme nous avons eu accès à un résultat d'examen en lien avec les deux cours durant lesquels les notes ont été prises, et ce, pour chaque participant ayant produit un document de notes, nous avons pu mettre en parallèle les indices de l'organisation du contenu des notes et de la sélection de ce contenu avec un résultat à un examen afin d'observer leur incidence sur la performance, et ce, dans les deux modes (objectif 1.2).

\section{Résultats}

\subsection{Organisation des notes (objectifs 1.1 et 1.1.1)}

Une grande différence dans la façon dont les noteurs organisent leurs notes est ressortie de notre analyse. Les notes manuscrites ne présentent qu'un seul type d'organisation tandis que les notes numériques en présentent trois.

Pour les notes manuscrites, les titres et les intertitres correspondent, en général, à des unités de sens des niveaux hiérarchiques supérieurs. Les noteurs font ressortir ces unités d'information grâce à diverses stratégies tout en rendant apparente la différence entre les titres et les intertitres : gras; soulignement ; ligne indépendante ; numérotation; alignement à gauche, à droite ou centré, etc. Pour ce qui est du traitement des unités de sens de niveaux hiérarchiques inférieurs, elles sont traitées comme du texte ou font partie de listes à puces. De plus, la mise en page semble uniforme, c'est-à-dire que les informations sont traitées de la même manière d'une page à l'autre des notes. En résumé, les noteurs prenant leurs notes à la main mettent bien en évidence la MS du DS dans leurs notes en différenciant les unités de sens de niveaux supérieurs (unités majeures et conceptuelles) des unités de sens de niveaux 
inférieurs (unités de sens de base et sous-unités de sens de base) par leur mise en forme. sens présentes dans les documents de notes manuscrites et numériques. Nous avons constaté que les noteurs prenant des notes manuscrites utilisent en moyenne $15,92$ unités de sens (ÉT = 5,93, IC à $95 \%=[12,15-19,68])$, ce qui est inférieur au nombre moyen utilisé par les noteurs prenant des notes numériques : 19,40 unités de sens (ÉT = 10,88, IC à $95 \%=[11,62-27,18])$. Cependant, un test statistique de différence (test de Student) a montré que la différence moyenne de $-3,48$ (IC à $95 \%=[-11,09-4,79]$ ) entre les modes manuscrit et numérique n'est pas significative $(t(13,34)=-0,907$, $p=0,381$ ) sur le plan statistique. Elle ne peut se généraliser à la population, mais elle n'en demeure pas moins présente dans l'échantillon.

\subsubsection{Sélection des unités de sens selon leur niveau hiérarchique}

De façon générale, nous constatons que les noteurs prenant des notes manuscrites procèdent à une meilleure sélection des informations que ceux prenant leurs notes numériques, mais la différence est assez minime. En effet, ils délaisseraient en moyenne plus d'informations que ceux prenant des notes numériques : $3 \%$ de sous-unités de base (unités les plus accessoires), $7 \%$ d'unités de base (unités accessoires) et $15 \%$ d'unités conceptuelles. Ils accorderaient toutefois autant d'importance aux unités majeures (unités essentielles) que ceux prenant des notes numériques en notant environ $50 \%$ de ces dernières. 


\subsubsection{Séquences du cours}

Pour ce qui est des séquences du cours, nous remarquons que les noteurs prenant des notes manuscrites et numériques ont noté la plupart des séquences théoriques, mais ont assez fréquemment omis de noter les séquences pratiques, surtout quand ces dernières étaient précédées de séquences théoriques, et ne servaient donc qu'à mettre en pratique la théorie vue au préalable. La plupart des noteurs ont toutefois noté la séquence pratique qui précédait la séquence théorique, car elle était en quelque sorte l'assise de la séquence théorique.

\subsection{Performance (objectif 1.2)}

Nous voulions voir si le mode de la PDN aurait une incidence sur les habitudes des noteurs, c'est-à-dire sur la façon dont ils organisent leurs notes et sélectionnent le contenu des notes, et si cela se répercuterait sur la performance à un examen.

Nous avons d'abord calculé la moyenne obtenue à l'examen par les noteurs des deux modes. Les 12 noteurs prenant leurs notes à la main ont obtenu un résultat moyen de $83,92 \%(E ́ T=6,49$, IC à $95 \%=[79,79-88,04])$ et les 10 noteurs prenant leurs notes à l'aide de la technologie ont obtenu un résultat moyen légèrement supérieur de 85,10\% (ÉT $=6,90$, IC à $95 \%=[80,16-90,03])$. Un test statistique de différence (test de Student) a montré que la différence moyenne de $-1,18$ (IC à $95 \%=[-7,15-4,78]$ ) entre les modes manuscrit et numérique n'est pas significative $(t(20)=-0,414, p=0,683)$ sur le plan statistique, ce qui signifie qu'elle ne peut être généralisée à la population. Il n'en demeure pas moins que cette différence a été constatée sur notre échantillon, mais reste très minime.

Par la suite, nous avons calculé la moyenne obtenue à l'examen selon le type d'organisation des notes. Pour le premier type d'organisation qui met bien en évidence la MS du DS et qui comprend tous les documents de notes manuscrites ainsi que 6 documents de notes numériques sur 10, les noteurs des deux modes ont eu un résultat moyen similaire : $83,92 \%$ pour les notes réalisées à la main et $84,92 \%$ pour les notes réalisées à l'aide de la technologie. Pour le deuxième type d'organisation qui ressemble à un amas d'informations très hiérarchisé, qui met moins bien en évidence la MS du DS et qui comprend 2 documents de notes numériques sur 10, les noteurs organisant leurs notes selon ce type n'ont pas reçu de résultat moyen bien différent de celui qu'ont reçu les noteurs organisant leurs notes selon le premier type : 86,75\% (ÉT =3,18). Il en est de même pour le troisième type d'organisation pour lequel 2 documents de notes numériques ont été réalisés par l'insertion de documents de référence sans l'ajout d'une hiérarchisation globale aux notes : $84 \%(E ́ T=14,14)$. Toutefois, il faut se montrer prudent avec ces deux derniers résultats, car la moyenne n'est pas une mesure de tendance très fiable étant donné la petite taille de l'échantillon, ce qu'il est possible de constater avec l'écart-type qui est parfois très important. Ainsi, en raison de la taille insuffisante des échantillons, il n'est pas clair si l'organisation des notes a eu une incidence sur la performance.

Pour ce qui est de la sélection du contenu des notes, un test de corrélation de Pearson entre le nombre d'unités de sens présent dans les notes et la performance a révélé une corrélation positive, c'est-à-dire que plus il y a d'unités de sens dans les notes, plus la 
performance est grande et vice-versa, mais elle n'est pas significative sur le plan statistique $(r=0,199, p=0,414)$.

De plus, il y aurait aussi une corrélation positive entre le nombre d'unités de sens de chacun des niveaux hiérarchiques présent dans les notes et le résultat à l'examen, mais, encore une fois, ces corrélations ne seraient pas significatives sur le plan statistique.

Enfin, pour ce qui est de la présence des séquences du cours dans les notes, en contexte d'examen à livre ouvert, la présence ou non de ces dernières dans les notes ne semble pas avoir une incidence sur la performance.

\section{Discussion}

\subsection{Limites}

Notre étude comporte certaines limites, liées notamment au contexte de la PDN des participants et à la taille de l'échantillon. Pour ce qui est du contexte de PDN, le fait que les participants aient eu accès à des documents de référence dans lesquels la MS du DS était en grande partie présente a fait en sorte que nous n'avons pas pu juger de la qualité des notes comme nous l'entendions, c'est-à-dire juger de la capacité du noteur à refléter lui-même la MS du DS dans ses notes. Nous avons plutôt observé l'organisation des notes et la sélection du contenu, mais il n'est pas clair si ces observations nous permettent vraiment d'évaluer la qualité des notes. De plus, disposant de ces documents, les étudiants ont probablement pris moins de notes que d'habitude. Par ailleurs, le contexte d'évaluation qui suivait ces prises de notes, examen à livre ouvert, rend difficile la mise en parallèle des notes et des résultats à l'examen, car il est impossible de savoir si la performance est attribuable uniquement aux prises de notes. Elle pourrait aussi être liée à l'accessibilité des documents de référence. Pour ce qui est de la taille de l'échantillon, celui-ci étant petit, il est difficile de tirer des conclusions ou de faire ressortir des tendances des données.

Ces deux raisons nous avaient incitées à envisager une deuxième, puis une troisième collecte de données. Malheureusement, la pandémie ayant forcé l'arrêt des cours, nous avons dû effectuer l'analyse sur les données de la première collecte.

\subsection{Interprétation des résultats}

Pour ce qui est de l'organisation des notes, nous avons mis en évidence le fait que la plupart des participants prenant des notes numériques utilisent la technologie pour prendre et organiser leurs notes de la même façon qu'ils le feraient à la main. Ce premier type d'organisation des notes permet de bien mettre en évidence la MS du DS. Cette constatation est à la fois intéressante et surprenante, car avec l'accès aux nombreuses fonctionnalités de la technologie, les étudiants seraient potentiellement en mesure de prendre leurs notes plus rapidement et plus efficacement en ajoutant, par exemple, un copier-coller, une vidéo enregistrée en classe, des schémas, etc. Cela fait surgir plusieurs questions : Connaissent-ils toutes les fonctionnalités offertes par la technologie pour la PDN? Savent-ils utiliser efficacement correctement la technologie ? Ont-ils reçu des formations à la PDN? Ont-ils reçu des formations pour utiliser la technologie, les logiciels ou les applications pour la PDN? Nous avons aussi mis en évidence que les noteurs numériques organisent leurs notes de deux autres 
façons : en plaçant les unités de sens en amas dans une liste à puces très hiérarchisée ou en insérant directement les documents de référence dans leurs notes sans les introduire ou les organiser. Il semble que dans les deux cas, les noteurs ont usé abusivement d'une seule fonctionnalité de la technologie, et ce, peut-être par manque d'expérience ou de compétence à prendre des notes numériques. Cela nous amène à nous poser les mêmes questions que celles mentionnées précédemment. En outre, dans les deux cas, l'organisation des notes ne permet pas de mettre la MS du DS en évidence. De plus, le deuxième type d'organisation, qui consiste à insérer des documents de référence, ne permet pas aux noteurs de profiter des bienfaits du processus de la prise de notes : la mémorisation, l'appropriation et la compréhension des informations qui mènent à la constitution d'une mémoire externe fiable et réutilisable (Piolat \& Boch, 2004).

Pour le contenu des notes, nous avons constaté que les noteurs numériques prennent en moyenne plus d'unités de sens en note que les noteurs manuscrits même si ce n'est pas significatif sur le plan statistique. Nous avons également relevé que les noteurs numériques semblent procéder à une moins bonne sélection des informations que les noteurs manuscrits, qui semblent prioriser la notation des unités de sens plus essentielles au détriment de celles plus accessoires. Cela concorde avec les écrits scientifiques qui relèvent que l'écriture numérique permet aux noteurs de noter plus d'informations (Aragón-Mendizábal et coll., 2016; Stacy \& Cain, 2015) de façon plus linéaire, comme un verbatim (Manzi et coll., 2017, p. 210), ce qui fait en sorte que les noteurs ont tendance à essayer de prendre toutes les informations en note sans les comprendre ou sélectionner celles qui sont plus importantes. L'écriture manuscrite, quant à elle, étant plus lente à cause des contraintes psychomotrices, obligerait les noteurs à sélectionner les informations à noter (Piolat, 2001) et donc à prendre le temps de les comprendre (Peverly \& Sumowski, 2012) et de se les approprier, ce qui permettrait un meilleur traitement de l'information dans la mémoire (Manzi et coll., 2017). Bref, ce ne serait pas la quantité d'unités de sens notées qui procurerait des bénéfices aux noteurs et qui aurait une incidence sur la mémorisation et ainsi sur la performance, mais plutôt la façon dont sont traitées les informations lors de la PDN.

Pour ce qui est de la performance à un examen, nous n'avons pas observé de différence entre les notes réalisées dans les deux modes en lien ou non avec l'organisation du contenu, la sélection de ce contenu ou la présence de certaines séquences du cours. Ceci est probablement attribuable à notre échantillon trop petit ou au contexte d'évaluation à livre ouvert. Toutefois, nous avons remarqué que pour deux questions sur une même notion, les étudiants prenant leurs notes de façon manuscrite ou numérique ont eu un meilleur résultat à la question théorique qu'à la question pratique. Pour la question théorique, ils ont eu un résultat moyen presque parfait et pour la question pratique, un résultat moyen un peu en dessous de $70 \%$. Nous avons alors émis l'hypothèse que les étudiants éprouvent peut-être de la difficulté à transférer les savoirs théoriques vers la pratique.

\section{Conclusion}

Au terme de cette recherche, nous ne pouvons pas dire si un mode ou l'autre permet de prendre des notes de meilleure qualité et si cela a une incidence sur la performance, mais nous avons remarqué que le mode numérique risque d'amener le noteur à utiliser 
les fonctionnalités de la technologie d'une façon qui pourrait se révéler moins efficace ou moins bénéfique en termes de mémorisation et de compréhension pour la PDN. D'autres recherches sont évidemment nécessaires pour confirmer (ou non) cette observation. Si tel est le cas, une telle observation pourra alors orienter la réflexion, dans les milieux scolaires et universitaires, au moins sur les deux points suivants: l'intégration en classe des technologies numériques pour faciliter la PDN ; la formation des apprenants à ces technologies (et, de manière plus générale, à la PDN). Il s'agira en fait de s'assurer que les apprenants utiliseront la technologie de façon judicieuse, efficace et qui se révèlera bénéfique pour l'activité de PDN.

Il est vrai qu'une autre collecte de données aurait probablement permis de mieux répondre à nos sous-objectifs de recherche. Nous avons donc fait des démarches auprès de plusieurs professeurs, avant le confinement lié à la Covid-19, pour trouver un contexte de collecte plus adéquat, c'est-à-dire un cours dans lequel les étudiants reflètent eux-mêmes la MS du DS dans leurs notes. Malheureusement, les professeurs rencontrés ont révélé qu'ils fournissaient la MS de leur cours aux étudiants dans des documents divers (Word, Powerpoint, etc.). Ils le font pour aider les étudiants qui éprouvent beaucoup de difficulté à prendre des notes parce qu'ils n'ont jamais appris à en prendre, ce qui a pour effet de ralentir la progression du cours.

Dans les prochaines recherches sur la PDN, nous croyons alors qu'il serait bon de s'intéresser aux difficultés que vivent les étudiants lors de leurs PDN en tenant compte de leur réalité à l'ère du numérique. De cette façon des pistes d'amélioration ou d'élaboration de formations à la PDN pourraient être proposées.

\section{BIBLIOGRAPHIE}

ARAGón-MendizÁbal, Estíbaliz, Delgado-CASAS, Cándida, NAVARRo-GuZMÁn, José-I., MENACHo-Jiménez, Inmaculada \& ROMERo-OlivA, Manuel-F. (2016). A Comparative Study of Handwriting and Computer Typing in Note-taking by University Students. Comunicar. Media Education Research Journal, 24(2). <www.scipedia.com/public/Aragon-Mendizabal_et_al_2016a>.

Восн, Françoise. (2000). Prise de notes et écriture conceptuelle à l'université. Pratiques, 105(1), 137-158. <https://doi.org/10.3406/prati.2000.2405>.

BOUCHET, Karine, Dufour, Sophie \& RENGIFO, Diana Lorena. (2014). Le pad : une aide à la prise de notes des étudiants allophones? <https://halshs.archives-ouvertes.fr/halshs-01376816/document>.

BRANCA-Rosoff, Sonia. (1998). Abréviations et icônes dans les prises de notes des étudiants. Dans M. Bilger, K. van den Eynde \& F. Gadet (dir.), Analyse linguistique et approches de l'oral : recueil d'études offert en hommage à Claire Blanche-Benveniste (p. 286-299). Peeters.

BUI, Dung C., Myerson, Joel \& HALE, Sandra. (2013). Note-taking with Computers: Exploring Alternative Strategies for Improved Recall. Journal of Educational Psychology, 105(2), 299-309. <https://doi.org/10.1037/a0030367>. 
CHAMBERLAND, Gilles, LAVOIE, Louisette \& MARQUIS, Danielle. (2011). 20 formules pédagogiques. Presses de l'Université du Québec.

Cody, Nadia, Coulombe, Sandra, Giroux, Patrick, Gauthier, Diane \& Gaudreault, Suzie. (2016).

Pratiques, objets et finalités de collaboration en lien avec l'intégration des tablettes numériques dans une école secondaire | Practices, Objects and Collaboration Purposes Related to the Integration of Digital Tablets in a High School. Canadian Journal of Learning and Technology, 42(3). <https://doi.org/10.21432/T2KK7S>.

FARACo, Martine, BARBIER, Marie-Laure, FALAISE, Achille \& BRANCA-Rosoff, Sonia. (2003). Codage et traitement automatique de corpus pour l'étude des prises de notes en français langue première et langue seconde. Arob@se,7(1-2), 97-117. <www2.lpl-aix.fr/ fulltext/1649.pdf>.

FIORELLA, Logan \& MAYER, Richard E. (2017). Spontaneous Spatial Strategy Use in Learning from Scientific Text. Contemporary Educational Psychology, 49, 66-79. <https://doi.org/10.1016/ j.cedpsych.2017.01.002>.

GOHIER, Christiane, CHEVRIER, Jacques \& ANADón, Marta. (2005). La formation des maitres au temps des réformes : l'identité professionnelle revisitée par la posture pédagogique. Dans D. Biron, M. Cividini \& J.-F. Desbiens (dir.), La profession enseignante au temps des réformes (p. 281-298). Éditions du CRP.

GOUVERNEMENT DU QUÉBEC. (2018). Plan d'action numérique en éducation et en enseignement supérieur. Ministère de l'Éducation et de l'Enseignement supérieur. <www.education.gouv.qc.ca/fileadmin/ site_web/documents/ministere/PAN_Plan_action_VF.pdf>.

KINTSCH, Walter \& VAN DIJK, Teun Adrianus. (1975). Comment on se rappelle et on résume des histoires. Langages, 9(40), 98-116. <https://doi.org/10.3406/lgge.1975.2300>.

LI, Siu Cheung \& Pow, Jacky W. C. (2011). Affordance of Deep Infusion of One-to-One Tablet-PCs into and beyond Classroom. International Journal of Instructional Media, 38(4), 319-326.

LONGCAMP, Marieke, BouCARD, Céline, GILHODES, Jean-Claude, ANTON, Jean-Luc, Roth, Muriel, NAZARIAN, Bruno \& VELAY, Jean-Luc. (2008). Learning through Hand- or Typewriting Influences Visual Recognition of New Graphic Shapes: Behavioral and Functional Imaging Evidence. Journal of Cognitive Neuroscience, 20(5), 802-815. <https://doi.org/10.1162/jocn.2008.20504>.

Luo, Linlin, KIEWRA, Kenneth, Flanigan, Abraham \& PeteraneTZ, Markeya. (2018). Laptop versus Longhand Note Taking: Effects on Lecture Notes and Achievement. Instructional Science, 46, 947-971. <https://doi.org/10.1007/s11251-018-9458-0>.

Manzi, Alberto, MartinEZ, Steven \& DurmysheVA, Yana. (2017). Cognitive Correlates of Lecture Note Taking: Handwriting Speed and Attention. North American Journal of Psychology, 19(1), 195-217.

MÉNARD, Louise. (2012). Apprentissage en classe et persévérance au premier cycle universitaire. Dans M. Romainville \& C. Michaut (dir.), Réussite, échec et abandon dans l'enseignement supérieur (p. 177-198). De Boeck Supérieur.

Mueller, Pam A. \& OpPenheImer, Daniel M. (2014). The Pen Is Mightier than the Keyboard: Advantages of Longhand over Laptop Note Taking. Psychological Science, 25(6), 1159-1168. <https://doi.org/10.1177/0956797614524581>.

PÉPIN, Audrey. (2020). Évaluation de la qualité des notes prises par des étudiants universitaires dans les modes numérique et manuscrit (Mémoire de maitrise). Université du Québec à Chicoutimi, Chicoutimi. <https://constellation.uqac.ca/5993/>. 
PÉPIN, Audrey, GAGnon, Odette, GIRoux, Patrick, Cody, Nadia, Coulombe, Sandra \& GAUTHIER, Diane. (2017). Prise de notes numériques au secondaire: comparaison de la qualité des notes numériques avec celle des notes manuscrites. Dépôt institutionnel de l'UQAC. <https://constellation.uqac.ca/4343/>.

PeVerLy, Stephen T. \& Sumowski, James F. (2012). What Variables Predict Quality of Text Notes and Are Text Notes Related to Performance on Different Types of Tests? Applied Cognitive Psychology, 26(1), 104-117. <https://doi.org/10.1002/acp.1802>.

Piolat, Annie. (2001). La prise de notes (1 $1^{\text {re }}$ éd.). Presses universitaires de France.

Piolat, Annie. (2010). Approche cognitive de la prise de notes comme écriture de l'urgence et de la mémoire externe. Le français d'aujourd'hui, 170(3), 51-62.

Piolat, Annie \& Boch, Françoise. (2004). Apprendre en notant et apprendre à noter. Dans É. Gentaz \& P. Dessus (dir.), Comprendre les apprentissages. Psychologie cognitive et éducation (p. 133-152). Dunod.

POLLET, Marie-Christine. (2001). Pour une didactique des discours universitaires. Étudiants et système de communication à l'université. De Boeck Supérieur. <https://doi.org/10.3917/dbu.polle.2001.01>.

PRÉGENT, Richard, BERNARD, Huguette \& KozANITIS, Anastassis. (2009). Enseigner à l'université dans une approche-programme : guide à l'intention des nouveaux professeurs et chargés de cours. Presses internationales Polytechnique.

Rebeyrolle, Josette, JACques, Marie-Paule \& PÉry-Woodley, Marie-Paule. (2009). Titres et intertitres dans l'organisation du discours. Journal of French Language Studies, 19, 269-290.

RINCK, Fanny \& MANSOUR, Léda. (2013). Littératie a l'ère du numérique : le copier-coller chez les étudiants. Ling. (dis)curso, 13(3), 613-637. <https://doi.org/10.1590/S1518-76322013000300007>.

RousseY, Jean-Yves \& Piolat, Annie. (2003). Prendre des notes et apprendre. Effet du mode d'accès à l'information et de la méthode de prise de notes. Arob@se, 7(1-2), 47-68.

STACY, Elizabeth Moore \& CAIN, Jeff. (2015). Note-taking and Handouts in The Digital Age. American Journal of Pharmaceutical Education, 79(7), 1-6. <https://doi.org/10.5688/ajpe797107>.

VAN DIJK, Teun Adrianus \& KINTSCH, Walter. (1983). Strategies of Discourse Comprehension. Academic Press. <www.discourses.org/OldBooks/Teun A van Dijk \& Walter Kintsch - Strategies of Discourse Comprehension.pdf>.

WiLLIAMS, Robert L. \& EGGERT, Alan C. (2002). Notetaking in College Classes: Student Patterns and Instructional Strategies. The Journal of General Education, 51(3), 173-199. <https://doi.org/10.1353/ jge.2003.0006>.

\section{ANNEXES}


Annexe I. - Grille d'analyse de la qualité des notes.

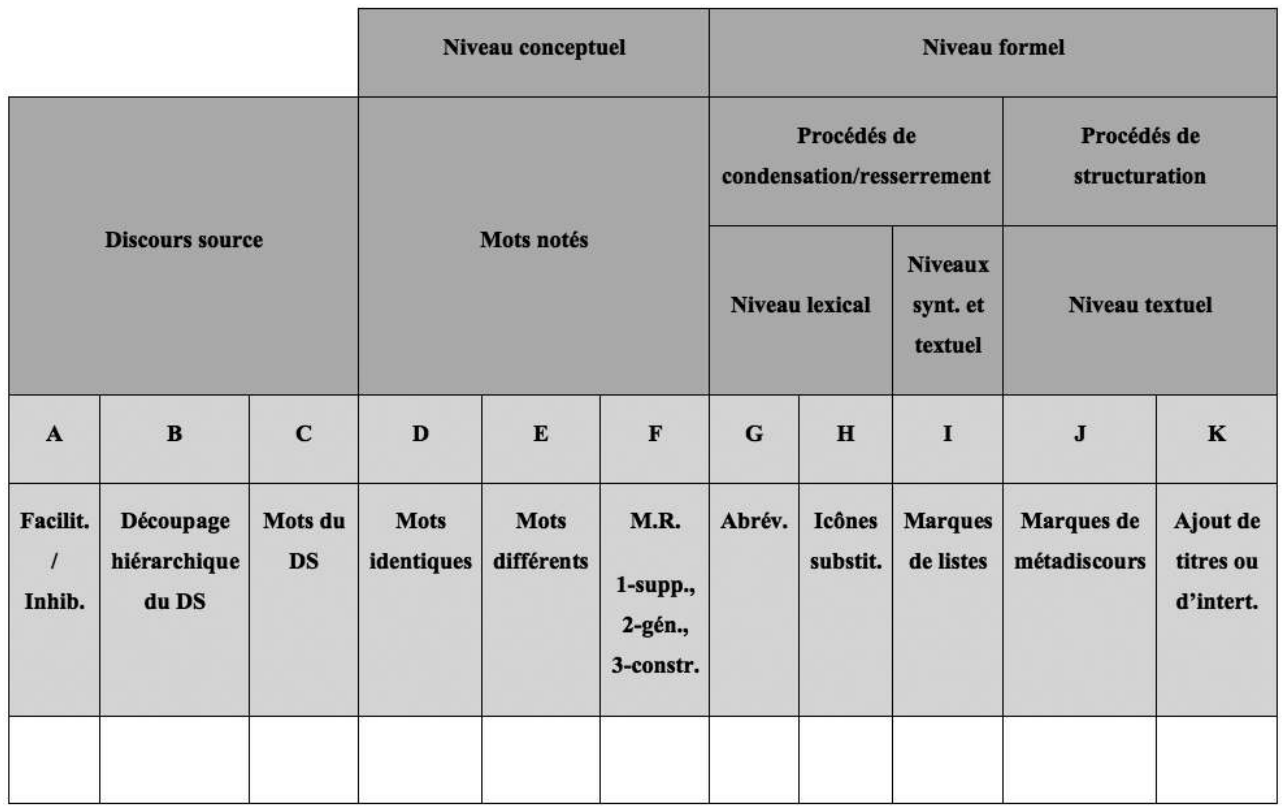

Annexe II. - Grille d'analyse de la qualité des notes modifiée.

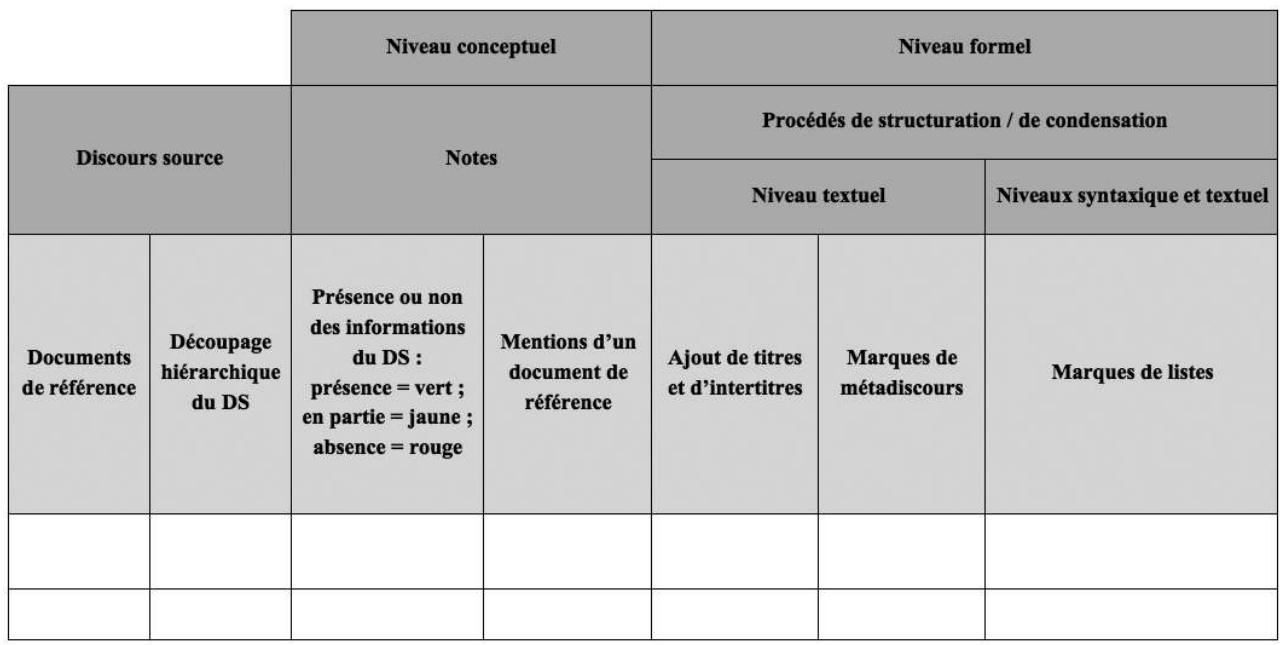

\section{NOTES}

1. Cette recherche a fait l'objet d'un mémoire de maitrise en linguistique à l'Université du Québec à Chicoutimi et la coauteure a bénéficié des bourses du Conseil de recherche en sciences humaines du Canada (CRSH) et du Fonds de recherche du Québec - Société et culture (FRQSC) pour ses travaux de recherche.

2. Cette grille n'a pas pu être utilisée telle quelle pour mesurer la qualité des notes de notre corpus comme nous l'entendions. En effet, il a fallu que nous modifiions notre grille d'analyse initiale afin de l'adapter aux caractéristiques de notre corpus. Nous en reparlerons à la $4^{\mathrm{e}}$ section. 3. Il est important de noter que cette grille est l'outil d'analyse du corpus et que la réalisation des autres objectifs est conditionnelle à la conception de cette grille, donc à la réalisation de ce premier objectif. 
4. Par souci de concision, ces trois macrorègles de réduction de l'information sémantique ne seront pas présentées dans l'article, le but ici étant de montrer ce dont nous avons tenu compte dans l'élaboration de notre grille d'analyse de la qualité des notes. Toutefois, pour plus de détails sur ces dernières, il est possible de consulter les références suivantes : Kintsch et Van Dijk (1975); Van Dijk et Kintsch (1983).

5. Il est important de noter que ce sont des exemples de présentation des informations selon les différents niveaux hiérarchiques. Il est possible que les noteurs utilisent d'autres manières de faire pour différencier les niveaux d'informations.

6. Le baccalauréat québécois est équivalent à la licence française.

7. Nous voulions procéder à une nouvelle collecte de données, mais avec le contexte de la pandémie, cela s'est avéré impossible.

8. Nous nous sommes également servies des notes que nous avons prises lorsque nous avons assisté aux deux séances de cours et des notes de la chargée de cours concernant la planification des deux cours pour faire le découpage hiérarchique du DS.

\section{RÉSUMÉS}

Avec l'intégration de plus en plus répandue de la technologie en classe, les apprenants voient leurs habitudes quant à la réalisation de diverses activités en classe modifiées à cause de l'affordance des technologies. Cette étude s'est intéressée plus spécifiquement aux notes réalisées dans les modes manuscrit et numérique, le but étant d'observer si l'affordance du mode, autrement dit les fonctionnalités offertes aux étudiants dans les deux modes, a un impact sur les habitudes des noteurs et sur la qualité des notes prises, et si cette dernière a un impact sur la performance à un examen. Pour nous, des notes de qualité sont des notes qui reflètent la macrostructure (MS) d'un discours source (DS). L'analyse de 10 documents de notes numériques et de 12 documents de notes manuscrites a notamment révélé qu'environ la moitié des étudiants prenant des notes numériques ont organisé leurs notes d'une façon qui ne permettait pas de faire ressortir la MS du DS tandis que tous les étudiants prenant des notes manuscrites ont réussi à le faire. Pour ce qui est du contenu des notes, tous les étudiants prenant des notes numériques ont moins bien sélectionné les informations qu'ils ont notées que ceux prenant des notes manuscrites. Il est toutefois impossible de dire si les différences dans la façon dont les notes ont été produites dans les deux modes a eu un impact sur la performance.

With the increasingly widespread integration of technology into the classroom, learners are seeing their habits of performing various activities in the classroom changed due to the overwhelming technology. This study was more specifically interested to note taking made in the manuscript and digital modes, but being to observe whether the affordance of the mode, in other words, the offers offered to students in both modes, have an impact on the habits of raters, and the quality of the notes taken, and whether this has an impact on performance on an exam. For us, quality notes are notes that reflect the macrostructure (MS) of a source speech (SS) In particular, analysis of 10 digital notes documents and 12 handwritten notes documents showed that about half of the students taking digital notes organized their notes in a way that did not bring out the MS of the SS while that all the students taking handwritten notes succeeded in doing so. In terms of notes content, all students taking digital notes were less successful in selecting the information they wrote down than those taking handwritten notes. It is, however, 
impossible to say whether the differences in the way the notes were generated in the two modes had an impact on the performance.

INDEX

Keywords : note-taking, macrostructure, digital mode, manuscript mode, performance, organization, selection of information

Mots-clés : prise de notes, macrostructure, mode numérique, mode manuscrit, performance, organisation, sélection des informations

\section{AUTEURS}

\section{AUDREY PÉPIN}

Doctorante en éducation, Université du Québec à Chicoutimi audrey.pepin1@uqac.ca

\section{ODETTE GAGNON}

Professeure, Université du Québec à Chicoutimi

odette_gagnon@uqac.ca 